\title{
A Fast-Acting Diagnostic Algorithm of Insulated Gate Bipolar Transistor Open Circuit Faults for Power Inverters in Electric Vehicles
}

\author{
Lei Yu ${ }^{1}$, Youtong Zhang ${ }^{1, *}$, Wenqing Huang ${ }^{2}$ and Khaled Teffah ${ }^{1}$ \\ 1 Laboratory of Low Emission Vehicle, Beijing Institute of Technology, Beijing 100081, China; \\ yulei0724@bit.edu.cn (L.Y.); baronecrist@hotmail.fr (K.T.) \\ 2 United Automotive Electronic System Co., Ltd., Shanghai 201206, China; hwq086@126.com \\ * Correspondence: youtong@bit.edu.cn; Tel.: +86-10-6891-5013
}

Academic Editor: K.T. Chau

Received: 20 January 2017; Accepted: 10 April 2017; Published: 18 April 2017

\begin{abstract}
To improve the diagnostic detection speed in electric vehicles, a novel diagnostic algorithm of insulated gate bipolar transistor (IGBT) open circuit faults for power inverters is proposed in this paper. The average of the difference between the actual three-phase current and referential three-phase current values over one electrical period is used as the diagnostic variable. The normalization method based on the amplitude of the $d-q$ axis referential current is applied to the diagnostic variables to improve the response speed of diagnosis, and to avoid the noise and the delay caused by signal acquisition. In the parameter discretization process, the variable parameter moving average method (VPMAM) is adopted to solve the variation of the average value over a period with the speed of the motor; hence, the diagnostic reliability of the system is improved. This algorithm is robust, independent of load variations, and has a high resistivity against false alarms. Since only the three-phase current of the motor is utilized for calculations in the time domain, a fast diagnostic detection speed can be achieved, which is significantly essential for real-time control in electric vehicles. The effectiveness of the proposed algorithm is verified by both simulation and experimental results.
\end{abstract}

Keywords: open circuit fault; insulated gate bipolar transistor (IGBT); inverter; electric vehicle

\section{Introduction}

With the advent of more stringent regulations related to emissions, fuel economy, and global warming, as well as energy resource constraints, electrical, hybrid, and fuel-cell vehicles have attracted increasing attention from vehicle constructors, governments, and consumers [1]. Electrical machines and drives are a key enabling technology for electrical, hybrid, and fuel cell vehicles [2]. The fault diagnosis of rotating electrical machines has received an intense amount of research interest during the last 30 years [3]. The failure of inverters is fatal in electric vehicles. Therefore, it is vital to adopt various algorithms to improve the diagnosis of the inverter faults. Timely maintenance and repair after a failure is very important to prevent a secondary failure, and to ensure the safe and reliable operation of the control system in electric vehicles.

Short-circuit faults and open circuit faults are the most common faults of insulated gate bipolar transistors (IGBTs) in power inverters [4]. Short-circuit faults are generally protected by hardware for its short maintenance time which is generally less than $10 \mu \mathrm{s}$. When open circuit faults occur, the motor is still able to operate. A new secondary failure may occur if it is not detected in the meanwhile. During driving, these faults may reduce safety which is fatal for electric vehicles. Therefore, it is essential to detect the open circuit faults in time. 
According to the classification of diagnostic signals, the existing diagnostic algorithms for IGBT open circuit faults can be broadly divided into the current-based diagnostic algorithms and the voltage-based diagnostic algorithms. Since the voltage source inverter outputs the voltage signals, the voltage-based diagnostic algorithms can quickly reflect the current status of the power switch and have a high reliability. Fast detection times can be achieved by using voltage-based algorithms [5-7]. A very fast detection scheme is proposed for the conventional three-leg converter that minimizes the use of voltage sensors in [8]. Faults and their locations are detected in less than $50 \mu \mathrm{s}$ for the studied parameters.

However, additional voltage sensors may be required, which increases the drive costs and complexity. Furthermore, in order to avoid false alarms, some time-delay values must be correctly defined, which can be very difficult since they depend on several variables [9]. Therefore, the voltage-based open circuit fault diagnostic algorithms are rarely used in electric vehicles. Current-based fault diagnostic algorithms do not need to add additional sensors. However, they have a certain response time constant because the current signal is a state parameter. Therefore, the current-based fault diagnostic algorithms are influenced by the system state and they have a certain time lag of detecting the faults.

For electric vehicles, in order to reduce the cost and complexity of the diagnostic system and ensure their safe and reliable operation, it is required to detect the open circuit faults as fast as possible while the diagnostic results need to be highly reliable without additional sensors [10].

An open circuit fault diagnostic algorithm based on the Park's vector center is proposed in [11]. In this algorithm, open circuit faults are detected by calculating the position of the current trajectory's midpoint, which is the average value of the AC current space vector over one period. An open circuit fault diagnosis algorithm based on Park's vector slope is proposed in [12]. The slope of the diameter of the current space vector trajectory is used to identify the faulty leg and the missing half-cycle of the current waveform is used to localize the faulty switch. In order to solve the drawback of being load dependent, a normalized DC current algorithm is proposed in [13], which is an improvement over Park's vector method. A modified normalized DC current algorithm is proposed in [14]. To prevent multiple satisfactory conditions, a less restrictive method is employed to localize the faulty switch. A fault detection and the improved fault-tolerant control for an open fault in the five-phase inverter driving system is proposed in [15]. A new fault diagnostic algorithm named the wind speed based normalized current trajectory is proposed and used to accurately detect and locate faulted IGBT in the circuit arms [16]. A very fast and efficient field-programmable gate array (FPGA) based on the open circuit switch fault detection method associated to fault tolerant converter topology in DC-DC converters is proposed in [17]. The detection time of the fault detection method is equal to or less than one switching period. Intelligent algorithms such as the clustering adaptive neuron fuzzy inference system [18], fuzzy-based algorithm [19], and wavelet transforms and neural network algorithms [20] are also proposed. However, due to the complexity of the intelligent algorithm and the large amount of computation, it is not suitable for real-time applications.

The fault diagnostic algorithms are compared and analyzed for their effectiveness, resistivity against false alarms, detection time, implementation, and tuning effort [21]. Among the open circuit fault algorithms, the modified normalized DC current algorithm is found to be very effective in detecting faults with a high resistivity to false alarms. In addition, it is independent of load variations. However, the modified normalized DC algorithm requires the discrete Fourier transform of the three-phase current, which may reduce the diagnostic detection speed.

The primary objective of this paper is to present a fast-acting diagnostic algorithm of IGBT open circuit faults for power inverters to improve the diagnostic detection speed, while ensuring the reliability of the diagnostic results in electric vehicles. The average of the difference between the actual three-phase current and the referential three-phase current values over one electrical period is used as the diagnostic variable. The three-phase referential current is obtained by transforming the target $d-q$ axis current. In order to eliminate the diagnostic error caused by the load variations, 
the normalization method is adopted. Since the magnitude of the $d-q$ axis current is the same as the magnitude of the three-phase current and is not affected by the signal acquisition, the average value of the $d-q$ axis current amplitude over one electrical period is taken as the normalized referential value. In the parameter discretization process, the variable parameter moving average method (VPMAM) is used to automatically adjust the counting cycle according to the motor speed to be adapted to the fault diagnosis at different motor speeds. As only the three-phase current is used, the system does not need additional sensors, which is beneficial regarding the system complexity and cost. This algorithm has the advantages of fast detection speed and high resistivity against false alarms which are significantly essential for real-time control in electric vehicles.

MATLAB/SIMULINK (MathWorks, Natick, MA, USA) models are built to analyze the proposed algorithm. Furthermore, experiments are carried out to verify the algorithm.

\section{Analysis of IGBT Open Circuit Faults}

The structure of the three-phase inverter is shown in Figure 1, where the six half-bridges corresponding to the six IGBTs are numbered T1-T6.

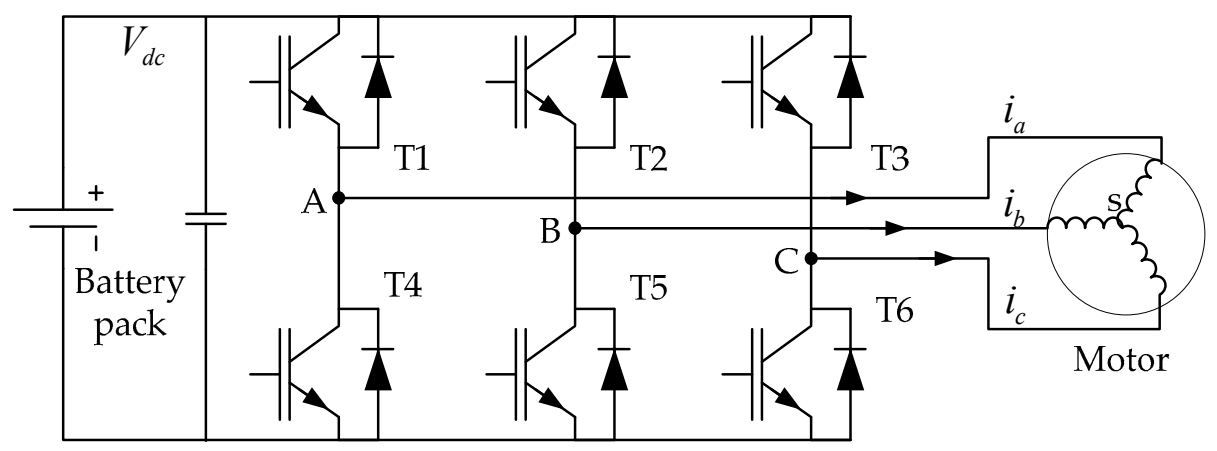

Figure 1. Structure of the three-phase inverter.

The current of phase A with an open circuit fault in T1 at $1000 \mathrm{rpm} / 100 \mathrm{Nm}$ is shown in Figure 2. In normal operation, by the regulation of the motor current closed-loop, the actual and referential three-phase currents of the motor are basically coincident. When an open circuit fault occurs in T1 at $0.05 \mathrm{~s}$, phase A cannot output positive voltage, and then the current regulator can only output negative current, leading to the difference between the actual and referential three-phase current.

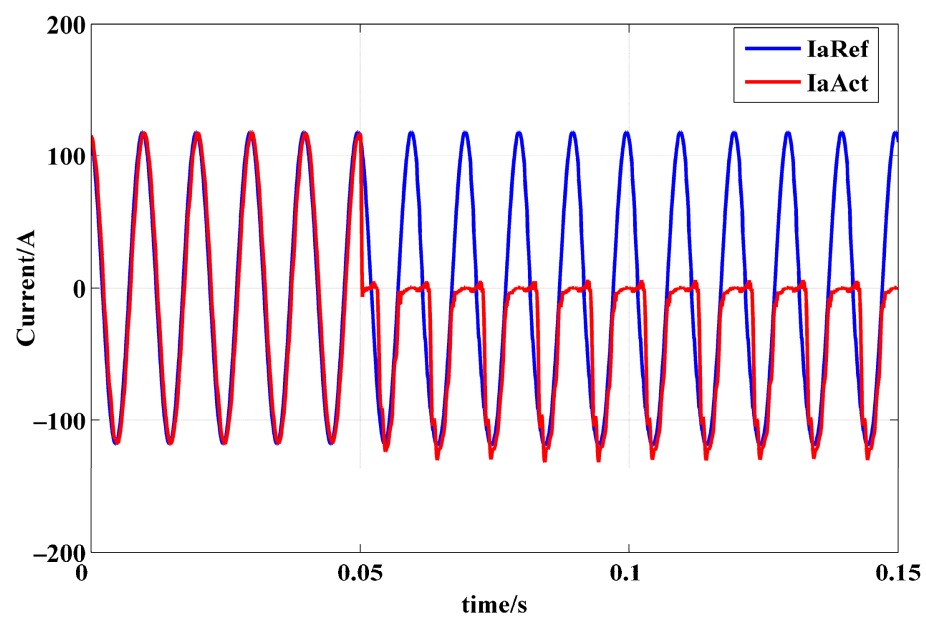

Figure 2. The current of phase A with an open circuit fault in T1 at $1000 \mathrm{rpm} / 100 \mathrm{Nm}$. 
Due to the three-phase symmetry, the trajectory of the stator current in normal operation is circular. The trajectory of the stator current at $1000 \mathrm{rpm} / 100 \mathrm{Nm}$ is shown in Figure 3.

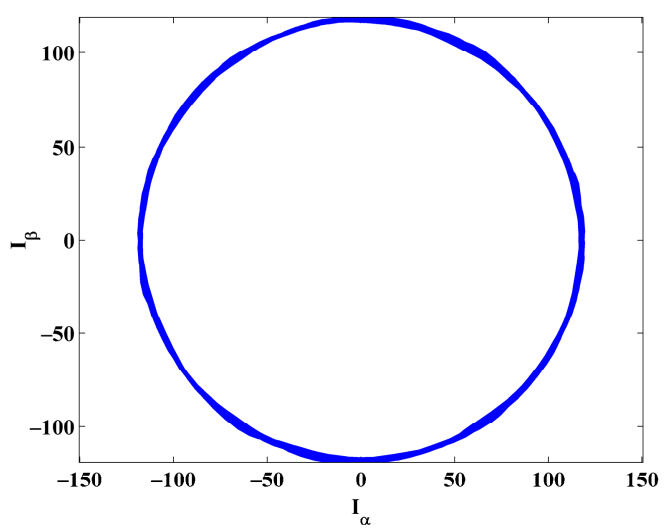

Figure 3. Trajectory of the current with no fault at $1000 \mathrm{rpm} / 100 \mathrm{Nm}$.

When an open circuit fault occurs, the trajectory of the stator current will be distorted, resulting in asymmetry, as shown in Figure 4. A trajectory similar to a straight line appears in the trajectory of the stator current. The trajectory appears only on one side of the circle, and it is no longer symmetrical.

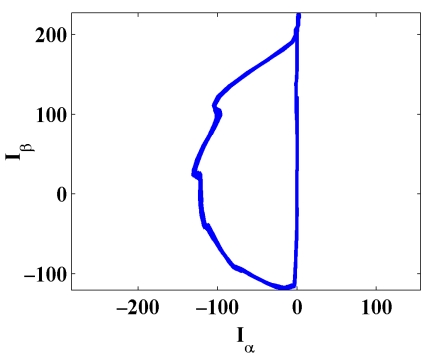

(a)

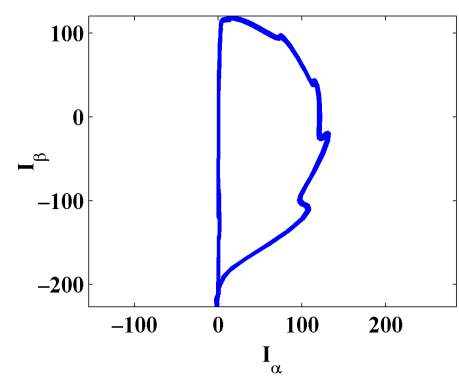

(d)

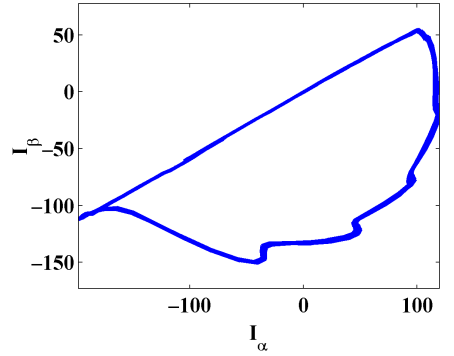

(b)

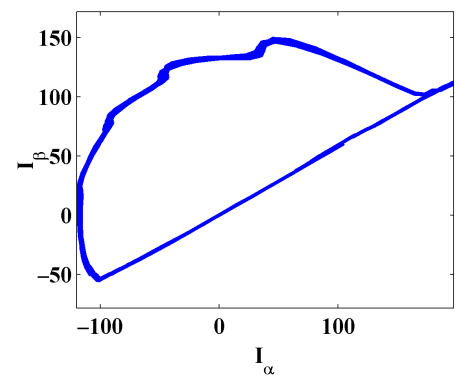

(e)

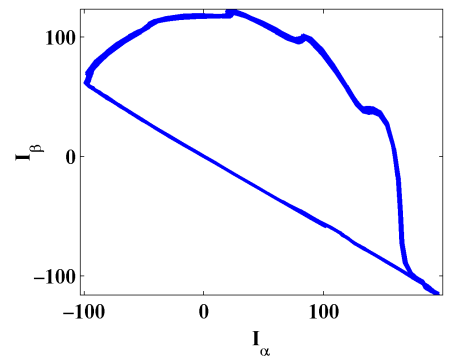

(c)

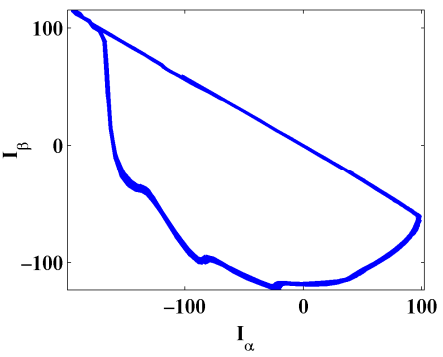

(f)

Figure 4. Trajectory of stator current with an open circuit fault at $1000 \mathrm{rpm} / 100 \mathrm{Nm}$ : (a) Open circuit fault in T1; (b) Open circuit fault in T2; (c) Open circuit fault in T3; (d) Open circuit fault in T4; (e) Open circuit fault in T5; and (f) Open circuit fault in T6.

\section{Proposed Fault Diagnostic Algorithm}

The proposed open circuit fault diagnostic algorithm is shown in Figure 5. 


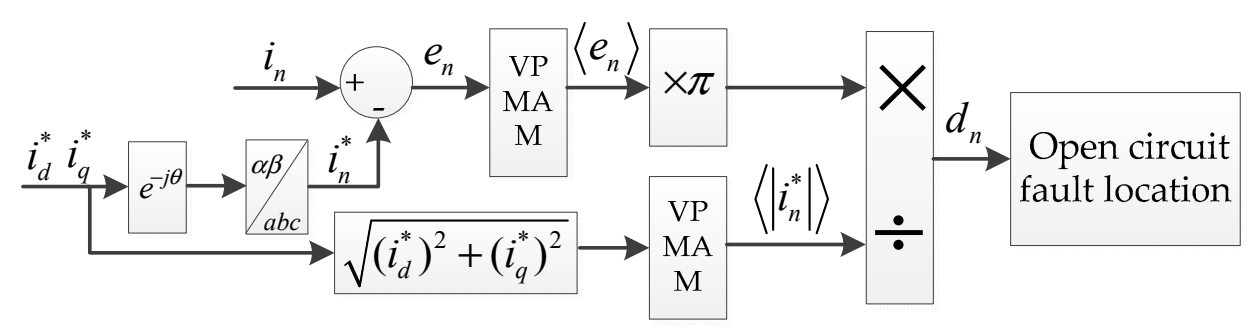

Figure 5. Diagram of the proposed open circuit fault diagnostic algorithm.

The $d-q$ axis current cannot be directly utilized for fault diagnosis because of the regulation of the current closed-loop. In this paper, the average of the difference between the actual three-phase current and the referential three-phase current values over one electrical period is used as the diagnostic variable. The three-phase referential current can be obtained from the target $d-q$ axis current through the inverse Park transformation and inverse Clark transformation.

The average value of parameter $X$ over one electrical period $\langle X\rangle$ can be expressed as follows:

$$
\langle X\rangle=f_{\text {current }} \int_{0}^{\frac{1}{f_{\text {current }}}} X \mathrm{~d} t=\frac{\omega_{e}}{2 \pi} \int_{0}^{\frac{2 \pi}{\omega_{e}}} X \mathrm{~d} t
$$

The difference between the actual three-phase current and the referential three-phase current values can be expressed as follows:

$$
e_{n}=i_{n}^{*}-i_{n}
$$

To improve the reliability of the diagnostic system, the average value of $e_{n}$ in one electrical period $\left\langle e_{n}\right\rangle$ is used as the diagnostic variable to reduce the misdiagnosis caused by the interference signal. The value of $\left\langle e_{n}\right\rangle$ is related to the magnitude of the phase current which represents the current load of the motor. In order to be independent of load variations, the normalization method is adopted. There are mainly three different normalized referential values: the first order harmonic coefficients of the three-phase current over one period [14]; the magnitude of the $d-q$ axis current over one period [11]; and the average absolute values of the three-phase current over one period [9]. The first order harmonic coefficients usually require a large amount of computation due to the utilization of the discrete Fourier transform. The average absolute values of the three-phase current are affected by the noise of the signal acquisition and signal delay. Since the amplitude of the $d-q$ axis current is the same as the magnitude of the three-phase current and is not affected by the signal acquisition, the $1 / \pi$ time of average amplitude of the $d-q$ axis current over one period is used as the normalized referential value. Thus, the normalized diagnostic variables can be expressed as follows:

$$
d_{n}=\frac{\pi\left\langle e_{n}\right\rangle}{\left\langle\sqrt{\left(i_{d}^{*}\right)^{2}+\left(i_{q}^{*}\right)^{2}}\right\rangle}
$$

Ideally, the three-phase current is fully balanced which can be expressed as follows:

$$
\begin{aligned}
& i_{a}=I_{m} \sin \left(\omega_{e} t+\phi\right) \\
& i_{b}=I_{m} \sin \left(\omega_{e} t-\frac{2 \pi}{3}+\phi\right) \\
& i_{c}=I_{m} \sin \left(\omega_{e} t-\frac{4 \pi}{3}+\phi\right)
\end{aligned}
$$

where $I_{m}$ is the amplitude of the three-phase current, $\phi$ is the initial phase, and $\omega_{e}$ is the electrical angular velocity. If the current follows this well, the referential $d-q$ axis current can be expressed as follows:

$$
\begin{aligned}
& i_{d}^{*}=I_{m} \cos \beta \\
& i_{q}^{*}=I_{m} \sin \beta
\end{aligned}
$$


where $\beta$ is the angle between the stator current vector and the $d$-axis.

The diagnostic variables can be obtained by substituting Equations (4) and (5) into Equations (1) and (3). Ideally, $i_{n}$ closely follows $i_{n}^{*}$ due to the current closed loop, and thus $e_{n}$ is equal to zero. Actually, $e_{n}$ is not equal to zero due to the acquisition error and noise. However, the average value of $e_{n}$ over one period $\left\langle e_{n}\right\rangle$ is approximately equal to zero due to the randomness of error. Therefore, $d_{n}$ is approximately equal to zero. When an open circuit fault occurs in T1, the upper half-bridge of phase A cannot output voltage. Therefore the output current of phase A in the first half of the period is zero, while the last half is normal. The output current of phase A can be expressed as follows:

$$
i_{a}= \begin{cases}0 & \frac{2 \pi-\phi}{\omega}<t<\frac{(2 k+1) \pi-\phi}{\omega} \\ I_{m} \sin (\omega t+\phi) & \frac{(2 k+1) \pi-\phi}{\omega}<t<\frac{(2 k+2) \pi-\phi}{\omega}\end{cases}
$$

Substituting Equation (6) into Equations (1) and (3), the diagnostic variable of phase A can be expressed as follows:

$$
d_{a}=\frac{\pi \times\left[\frac{\omega_{e}}{2 \pi} \int_{\frac{2 k \pi-\phi}{\omega_{e}}}^{\frac{(2 k+2) \pi-\phi}{\omega_{e}}} I_{m} \sin \left(\omega_{e} t+\phi\right) \mathrm{d} t-\frac{\omega_{e}}{2 \pi} \int \frac{\frac{(2 k+2) \pi-\phi}{\omega_{e}}}{\frac{(2 k+1) \pi-\phi}{\omega_{e}}} I_{m} \sin \left(\omega_{e} t+\phi\right) \mathrm{d} t\right]}{\frac{\omega_{e}}{2 \pi} \int_{\frac{2 \pi-\phi}{\omega_{e}}}^{\frac{(2 k+2) \pi-\phi}{\omega_{e}}}\left[\sqrt{\left(I_{m} \sin \beta\right)^{2}+\left(I_{m} \cos \beta\right)^{2}}\right] \mathrm{d} t}=1
$$

It can be similarly analyzed when the open circuit fault occurs in the other half-bridges. It can be proven that the value of the diagnostic variable $d_{n}$ is approximately equal to 1 for an open circuit fault in the upper half-bridge and -1 for an open circuit fault in the lower half-bridge. Therefore, the open circuit fault can be located by identifying which diagnostic variable is beyond the threshold $K_{f}$, as shown in Table 1.

Table 1. Localization of the open circuit fault. IGBT: Insulated Gate Bipolar Transistor.

\begin{tabular}{cccc}
\hline Faulty IGBT & $\boldsymbol{d}_{\boldsymbol{a}}$ & $\boldsymbol{d}_{\boldsymbol{b}}$ & $\boldsymbol{d}_{\boldsymbol{c}}$ \\
\hline $\mathrm{T} 1$ & $>K_{f}$ & - & - \\
$\mathrm{T} 2$ & - & $>K_{f}$ & - \\
$\mathrm{T} 3$ & - & - & $>K_{f}$ \\
$\mathrm{~T} 4$ & $<-K_{f}$ & - & - \\
$\mathrm{T} 5$ & - & $<-K_{f}$ & - \\
$\mathrm{T} 6$ & - & - & $<-K_{f}$ \\
\hline
\end{tabular}

Ideally, the values of the diagnostic variables are 0 and 1 in normal and faulty conditions, respectively. Therefore, $K_{f}$ should be between 0 and 1 . A smaller value of $K_{f}$ can improve the diagnostic speed of the system, but may also lead to the possibility of misdiagnosis and vice versa. In order to improve the system response speed and diagnostic reliability, the selection for the value of $K_{f}$ should consider the worst condition (usually a huge load variation) [9]. The value of $K_{f}$ should be as small as possible in the case of meeting the worst condition.

In the analysis above, $\langle X\rangle$ is defined as an analog parameter which needs to be discretized in the control process. The simple moving average method is commonly utilized to discretize the parameter. The simple moving average method averages a fixed number of data. It can be expressed as follows:

$$
X_{k-A v g}=\sum_{i=0}^{n-1} X((k-i) \tau) / n
$$

where $X_{k_{-} A v g}$ is the moving average value at k-time, $n$ is the number of data, $\tau$ is the control period, and $X((k-i) \tau)$ is the actual value at $\mathrm{i}$-time before $\mathrm{k}$-time. 
$\langle X\rangle$ is the average value of the discrete variables over an electrical period. However, the electrical period constantly changes with the motor speed. Therefore, the number of data used for the average cannot simply be a fixed value, it should vary with the electrical period synchronously. The VPMAM is obtained by replacing the parameter $n$ of the simple moving average method with the parameter $N$ which varies with the electrical period. $N$ can be expressed as follows:

$$
N=\frac{2 \pi}{\omega_{e} \tau}
$$

Thus, the VPMAM can be expressed as follows:

$$
\langle X\rangle=\frac{1}{N} \sum_{i=0}^{N-1} X((k-i) \tau)=\frac{\omega_{e} \tau}{2 \pi} \sum_{i=0}^{\frac{\omega_{e} \tau}{2 \pi}-1} X((k-i) \tau)
$$

\section{Simulation and Analysis}

MATLAB/SIMULINK models are developed to examine the proposed algorithm. The permanent magnet synchronous motor (PMSM) is an attractive candidate for electric vehicles due to its advantage of high power density [22]. A 75-kW PMSM for an electric vehicle is used in this paper, and its parameters are shown in Table 2 [23].

Table 2. Parameters of the Permanent Magnet Synchronous Motor (PMSM).

\begin{tabular}{ccc}
\hline Parameter & Symbol & Value \\
\hline Number of pole pairs & $p$ & 6 \\
Stator resistance & $R_{s}$ & $4.23 \mathrm{~m} \Omega$ \\
Magnet flux linkage & $\lambda_{s}$ & $0.1039 \mathrm{~Wb}$ \\
d-axis inductance & $L_{d}$ & $0.171 \mathrm{mH}$ \\
$q$-axis inductance & $L_{q}$ & $0.391 \mathrm{mH}$ \\
DC linkage voltage & $V_{d c}$ & $288 \mathrm{~V}$ \\
Maximum speed & $n_{b}$ & $4000 \mathrm{rpm}$ \\
Peak motor current & $I_{p k}$ & $570 \mathrm{~A}$ pk \\
Rated power & $P_{r}$ & $75 \mathrm{~kW}$ \\
Peak torque & $T_{\text {max }}$ & $540 \mathrm{Nm}$ \\
Coefficient of R Rac to $R_{\mathrm{sDC}}$ & $M$ & 0.1 \\
Hysteresis current coefficient & $K_{h}$ & 0.6637 \\
Eddy current coefficient & $K_{e}$ & 0.00084 \\
Stray loss coefficient & $c_{\text {Str }}$ & $2.56 \times 10^{-9}$ \\
Mechanical friction torque & $T_{\text {fric }}$ & 5.24 \\
Windage torque coefficient & $c_{\text {wind }}$ & $3.35 \times 10^{-3}$ \\
\hline
\end{tabular}

The simulation model is shown in Figure 6.

As shown in Figure 6, the simulation model includes the DC voltage source subsystem, the $75 \mathrm{~kW}$ PMSM subsystem, the universal bridge subsystem, and the PMSM-space vector modulation (PMSM-SVM) subsystem. Moreover, the proposed diagnostic algorithm is included in the PMSM-SVM subsystem. The pulse width modulation (PWM) frequency is set as $10 \mathrm{kHz}$ and the proportional integral (PI) current regulating frequency is set as $20 \mathrm{kHz}$. The dead time and the voltage drop of the power device are ignored in the simulation. The IGBT open circuit faults are generated by removing the gate command signals of the required IGBTs.

In order to illustrate that the proposed algorithm is independent of load variations, low speed $600 \mathrm{rpm}$, rated speed $2000 \mathrm{rpm}$, and high speed $2800 \mathrm{rpm}$ at rated torque conditions, rated speed at $10 \%$ rated torque, and sudden change of the load torque conditions are analyzed in this paper. Since the three phases are symmetrical, only the open circuit faults in phase A are analyzed. 


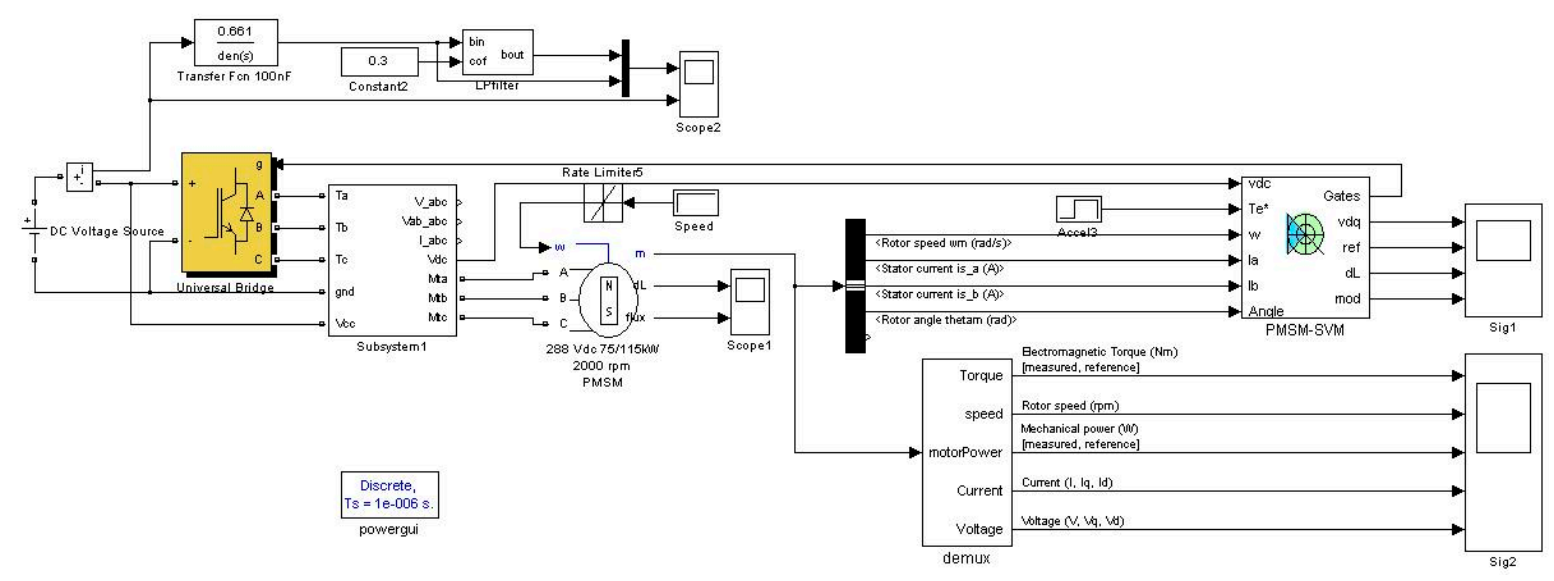

Figure 6. Simulation model.

\subsection{Analysis of the Response}

For convenience, the open circuit fault in the upper bridge of phase A is analyzed. Define $x$ as:

$$
x=\omega_{e} t+\phi
$$

Assuming that the open circuit fault occurs when $x=0$, the diagnostic variable $d_{a}$ changing with the rotor electrical angular degrees can be expressed as follows:

$$
d_{a}= \begin{cases}\frac{\pi\left[\int_{\theta-2 \pi}^{\theta} I_{m} \sin x \mathrm{~d} x-\left(-\int_{0}^{\theta} I_{m} \sin x \mathrm{~d} x\right)\right]}{\int_{\theta-2 \pi}^{\theta} I_{m} \mathrm{~d} x}=\frac{1-\cos \theta}{2} & \theta \leq \pi \\ 1 & \theta>\pi\end{cases}
$$

As shown in Equation (12), $d_{a}=0.5$ when $\theta=\pi / 2$, and $d_{a}=1$ when $\theta=\pi$. The diagnostic variable can reach 0.5 in one quarter of the electrical period and can reach 1 in half of the electrical period after the failure. Therefore, the detection speed is fast.

If the average absolute value of the actual three-phase current over one electrical period is used as the normalized referential value [9], the corresponding diagnostic variable $d_{a}^{\prime}$ can be expressed as follows:

$$
d_{a}^{\prime}= \begin{cases}\frac{\int_{\theta-2 \pi}^{\theta} \sin x \mathrm{~d} x-\left(-\int_{0}^{\theta} \sin x \mathrm{~d} x\right)}{\int_{\theta-2 \pi}^{\theta}|\sin x| \mathrm{d} x-\int_{0}^{\theta}|\sin x| \mathrm{d} x}=\frac{1-\cos \theta}{3+\cos \theta} & \theta \leq \pi \\ 1 & \theta>\pi\end{cases}
$$

The two diagnostic variables with different normalized referential values varying with rotor electrical angular degrees are plotted in Figure 7.

As shown in Figure 7, although the two diagnostic variables achieve stability at the same time, the algorithm presented in this paper has a higher value of the diagnostic variable and a better response before achieving stability. In fact, a value smaller than 1 is often used as a threshold considering the response. Compared with the algorithm using the actual current as the normalized referential value, the proposed algorithm can not only avoid the interference errors caused by the actual current acquisition, but also detect the faults faster. 


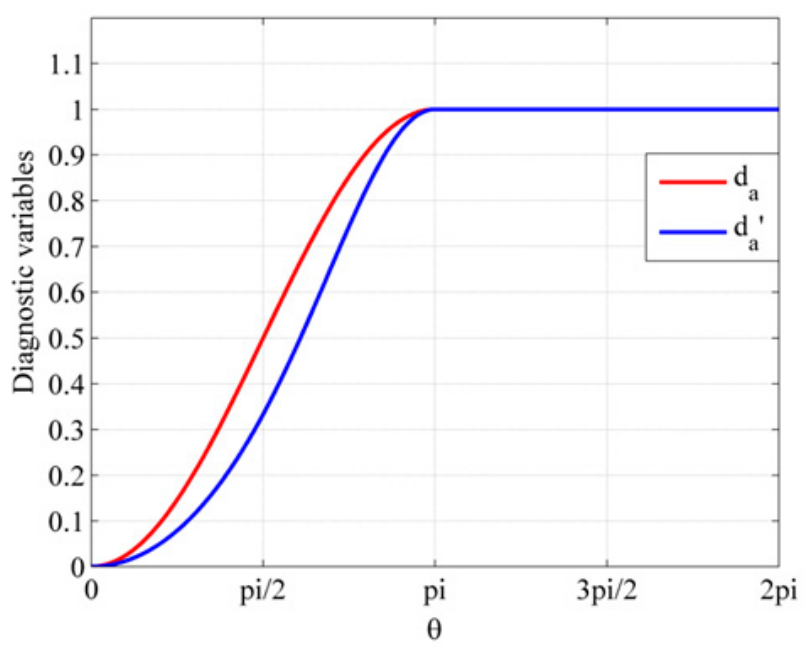

Figure 7. Comparison between the two diagnostic variables.

\subsection{Analysis of the Diagnostic Algorithm under Different Speed and Torque Conditions}

The simulation results when an open circuit fault occurs in T4 at $0.1 \mathrm{~s}$ at $2000 \mathrm{rpm} / 358 \mathrm{Nm}$ and $2000 \mathrm{rpm} / 36 \mathrm{Nm}$ are shown in Figures 8 and 9. As shown in Figure 8, at $2000 \mathrm{rpm} / 358 \mathrm{Nm}$, the output torque and current of the motor are stable and the diagnostic variable is around zero under normal conditions. When an open circuit fault occurs in T4, phase A can only output positive current, while the currents of phase $B$ and phase $C$ are distorted and fluctuate with the peak-to-peak value up to $1500 \mathrm{~A}$. Furthermore, the output torque of the motor is unstable, the period of the torque ripple is the same as the period of the phase current, and the peak-to-peak value of the torque ripple is above $800 \mathrm{Nm}$. The diagnostic variables change rapidly after the open circuit fault occurs. The value of $D a$ decreases to approximately 1.05 (existing error due to the white noise during current sampling in the simulation) and the other two variables of $D b$ and $D c$ increase to approximately 0.34 and 0.22 . Furthermore, when the current of phase $\mathrm{A}$ is in the upper half period at $0.1 \mathrm{~s}$, the open circuit fault does not affect the actual current. When the current of phase A crosses zero at $0.1011 \mathrm{~s}$, and since phase A cannot output negative voltage, the three-phase current starts to distort due to the open circuit fault. The value of $D a$ decreases to -0.5 and -1 at $0.1023 \mathrm{~s}$ (after $46 \%$ of the current period) and $0.1031 \mathrm{~s}$ (after $62 \%$ of the current period), respectively. At $2000 \mathrm{rpm} / 36 \mathrm{Nm}$, the stable value of the three diagnostic variables are $-1,0.44$ and -0.16 , respectively. The simulation results are similar to those at $2000 \mathrm{rpm} / 358 \mathrm{Nm}$ except for the magnitude of the torque and current.

The simulation results when an open circuit fault occurs in T4 at $0.1 \mathrm{~s}$ at $600 \mathrm{rpm} / 358 \mathrm{Nm}$ and $2800 \mathrm{rpm} / 256 \mathrm{Nm}$ are shown in Figures 10 and 11. As shown, the performance of the diagnostic algorithm at lower and higher speeds is consistent with that under rated condition. The open circuit fault can be detected accurately and the value of $D a$ is stable around -1 . The simulation results show that the proposed algorithm is independent of load variations and has a good robustness. 

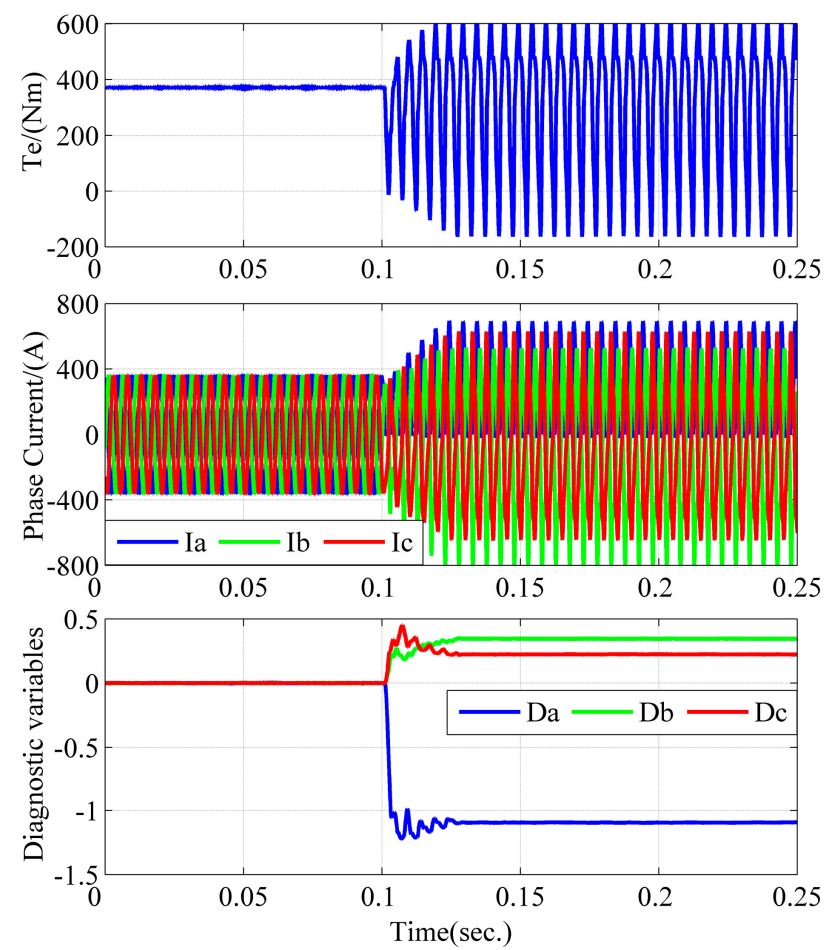

Figure 8. Diagnostic results at $2000 \mathrm{rpm} / 358 \mathrm{Nm}$.
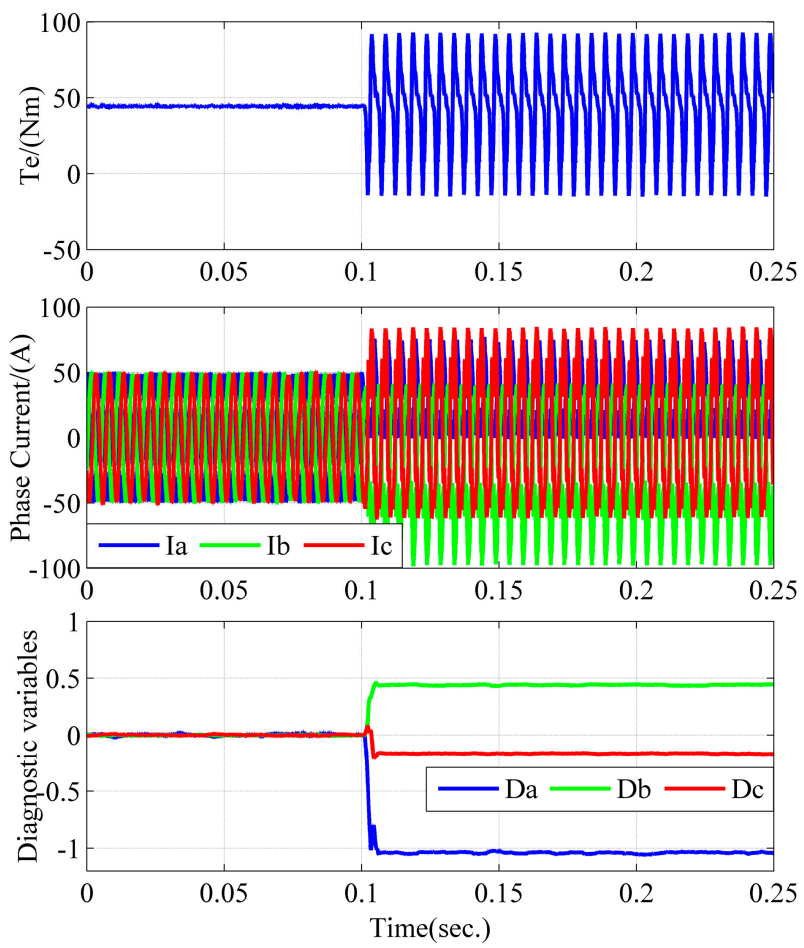

Figure 9. Diagnostic results at $2000 \mathrm{rpm} / 36 \mathrm{Nm}$. 

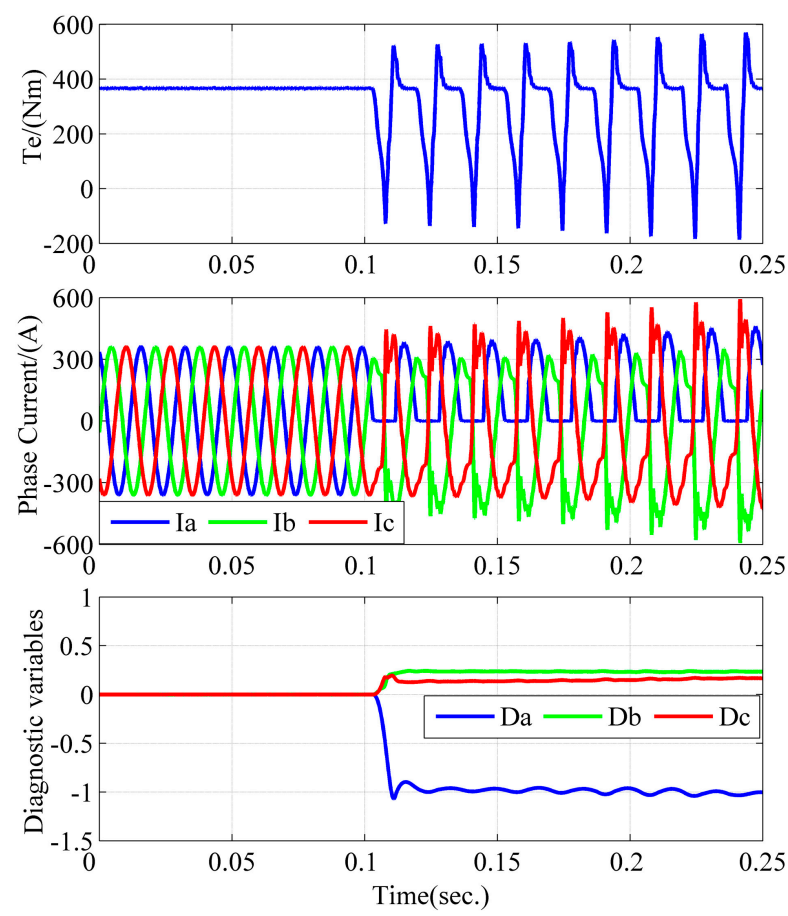

Figure 10. Diagnostic results at $600 \mathrm{rpm} / 358 \mathrm{Nm}$.
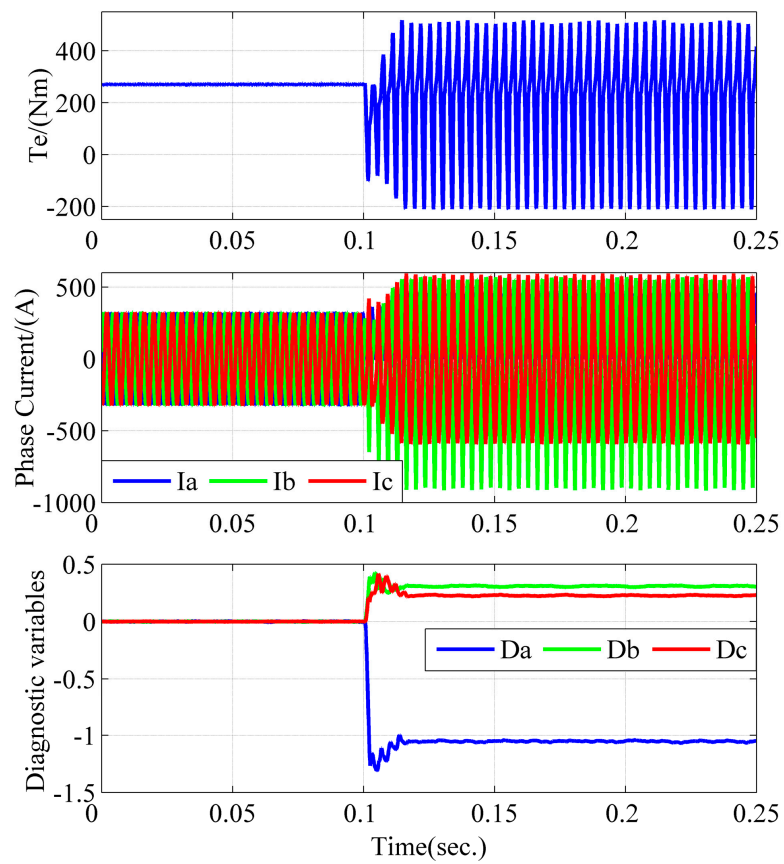

Figure 11. Diagnostic results at $2800 \mathrm{rpm} / 256 \mathrm{Nm}$.

\subsection{Analysis of the Diagnostic Algorithm under a Sudden Change of Load Torque Conditions}

To illustrate the resistivity against false alarms of the proposed algorithm, a torque pulse from zero to the rated torque is applied to the motor at rated speed $(2000 \mathrm{rpm})$, and the simulation results are shown in Figure 12. When the load torque of the motor increases to the rated torque from zero at $0.01 \mathrm{~s}$, the difference between the actual three-phase current and the referential three-phase current values is slightly increased, leading to the increase of the diagnostic variables. When the load torque of the motor decreases to zero from the rated torque at $0.05 \mathrm{~s}$, the diagnostic variables also increase. 
However, as shown in Figure 12, the amplitudes of the diagnostic variables are always smaller than 0.5 under normal conditions.
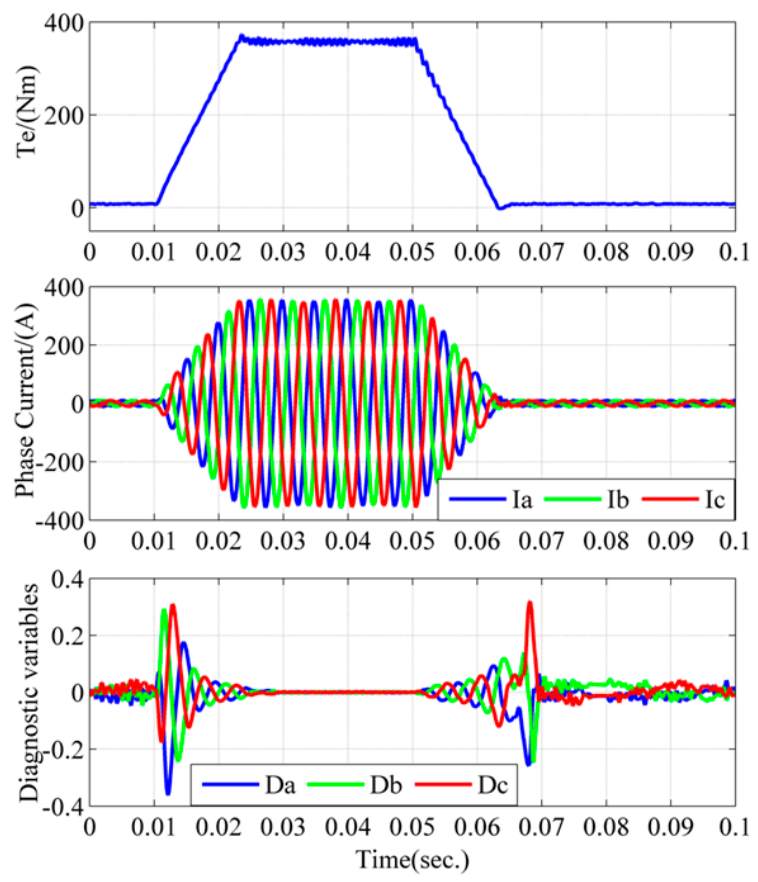

Figure 12. Diagnostic results under a sudden change of load torque conditions.

\section{Experimental Results}

The effectiveness of the proposed algorithm is tested experimentally with the PMSM as shown in Table 2. The experimental setup basically comprises a PMSM coupled to a Siemens dynamometer (1GP5-280) (Siemens Aktiengesellschaft, Munich, Germany) test system, a LiFePO4 battery pack (288 V/180 Ah), a HBM torque meter (T40) (Hottinger Baldwin Messtechnik GmbH, Darmstadt, Germany) and an inverter developed internally. In the inverter, an Infineon six-pack IGBT (FS800R07A2E3) (Infineon Technologies AG, Neubiberg, Germany) is used as the power switches; a TI digital signal processor (TMS320F28335) (Texas Instruments Incorporated, Dallas, TX, USA) carries out the real-time algorithm; three LEM current sensors (HAH1DR 900-s) (LEM Switzerland SA, Genève, Switzerland) measure the stator phase current. The experimental bench is shown in Figure 13.

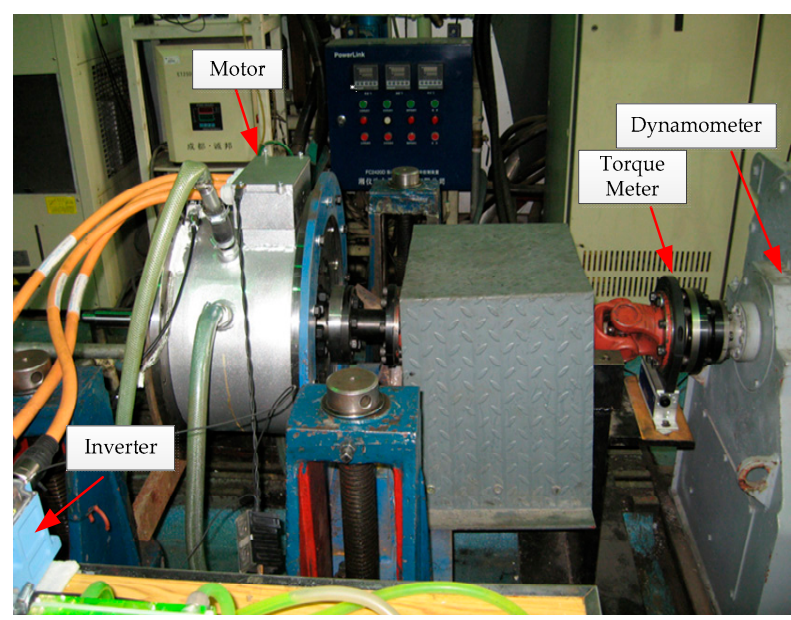

Figure 13. Experimental Bench. 
The IGBT open circuit faults are controlled by the user by removing the gate command signals of the required IGBTs. The maximum implemented collector current of the IGBT used in the experiments is $800 \mathrm{~A}$. Considering the reliability, the maximum amplitude of the phase current is limited to $600 \mathrm{~A}$ by the Altera complex programmable logic device (EPM240T100I5N) (Intel Corporation, Santa Clara, CA, USA). The three-phase current will be distorted in the fault state. It is difficult to obtain the experimental results at $2000 \mathrm{rpm} / 358 \mathrm{Nm}$ and $2800 \mathrm{rpm} / 256 \mathrm{Nm}$ because the distorted current will exceed the limited amplitude of the phase current. Therefore, the experiments are operated at $600 \mathrm{rpm} / 358 \mathrm{Nm}$ and under a sudden change of the load torque conditions. The experimental results are shown in Figures 14 and 15.

As shown in Figure 14, at $600 \mathrm{rpm} / 358 \mathrm{Nm}$, the output torque and current of the motor are stable and the diagnostic variables are around zero under normal conditions. When an open circuit fault occurs in T4 at $0.1 \mathrm{~s}$, phase A can only output positive current, while the currents of phase B and phase $\mathrm{C}$ are distorted and fluctuate with the peak-to-peak value up to $1200 \mathrm{~A}$. Furthermore, the output torque of the motor is unstable, the period of the torque ripple is the same as the period of the phase current, and the peak-to-peak value of the torque ripple is above $700 \mathrm{Nm}$. The diagnostic variables change rapidly after the open circuit fault occurs. The value of $D a$ decreases to approximately -1 and the other two variables of $D b$ and $D c$ increase to approximately 0.41 and 0.32 . Moreover, when the current of phase $\mathrm{A}$ is in the upper half period at $0.1 \mathrm{~s}$, the open circuit fault does not affect the actual current immediately. When the current of phase A is crossing zero at $0.1021 \mathrm{~s}$, and since phase A cannot output negative voltage, the three-phase current starts to distort due to the open circuit fault. The value of $D a$ decreases to -0.5 and -1 at $0.1045 \mathrm{~s}$ (after $27 \%$ of the current period) and $0.1047 \mathrm{~s}$ (after $28 \%$ of the current period), respectively.

To illustrate the resistivity against false alarms of the proposed algorithm, a torque pulse from zero to the rated torque is applied to the motor at rated speed $(2000 \mathrm{rpm})$, and the experimental results are shown in Figure 15. When the load torque of the motor increases to the rated torque from zero at $0.05 \mathrm{~s}$, the difference between the actual three-phase current and the referential three-phase current values increases slightly, leading to the increase of the diagnostic variables. When the load torque of the motor decreases to zero from the rated torque at $0.2 \mathrm{~s}$, the diagnostic variables also increase. However, as shown in Figure 15, the amplitudes of the diagnostic variables are always smaller than 0.5 under normal conditions. Considering the response and reliability of the system, the threshold $K_{f}$ is set to be 0.75 .

As shown in Figures 14 and 15, the output torque and the diagnostic variables have a certain lower harmonic. Under normal conditions, the three-phase current is not completely symmetrical, however, the trend is basically the same as that in the simulations. The reason of the deviation is that the simulation results do not take into account the dead-time effect of the switch, and the deviation and noise of the current and the position acquisition, resulting in the simulation results being better than the experimental results.

As shown in the simulation and experimental results, since the diagnostic variables are normalized, the thresholds are universal and can distinguish the open circuit faults accurately at different loads of the motor. Thus, this algorithm is independent of the load variations.

Typically, the detection speed of a current-based algorithm depends on the time instant of the fault occurrence [24]. Specifically, if a fault occurs in the bottom IGBT and during the negative current half-cycle of the respective phase, the effect can be immediately seen since the current tends to be zero. Thus, the detection speed is relatively faster. However, if a fault occurs in the bottom IGBT and during the positive current half-cycle of the respective phase, the fault effects cannot be immediately seen and will just be seen at the next current half-cycle; hence, the fault can remain undetected for a period of time that exceeds half of the current fundamental period. Thus, the detection speed is relatively slower. An equivalent result is also verified for a fault in the top IGBT of the respective phase. Considering this, the best way to evaluate the detection speed is to obtain the detection time for a fault occurrence at different instants of the current period. 

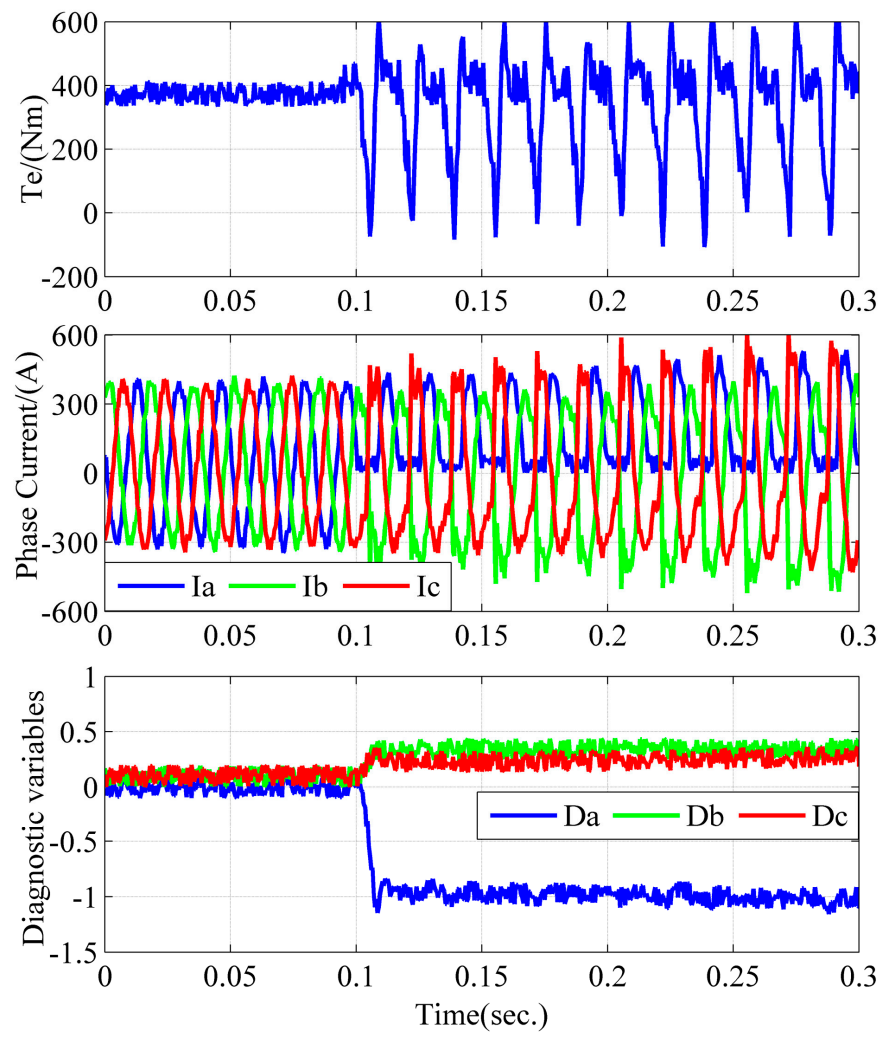

Figure 14. Experimental results at $600 \mathrm{rpm} / 358 \mathrm{Nm}$.
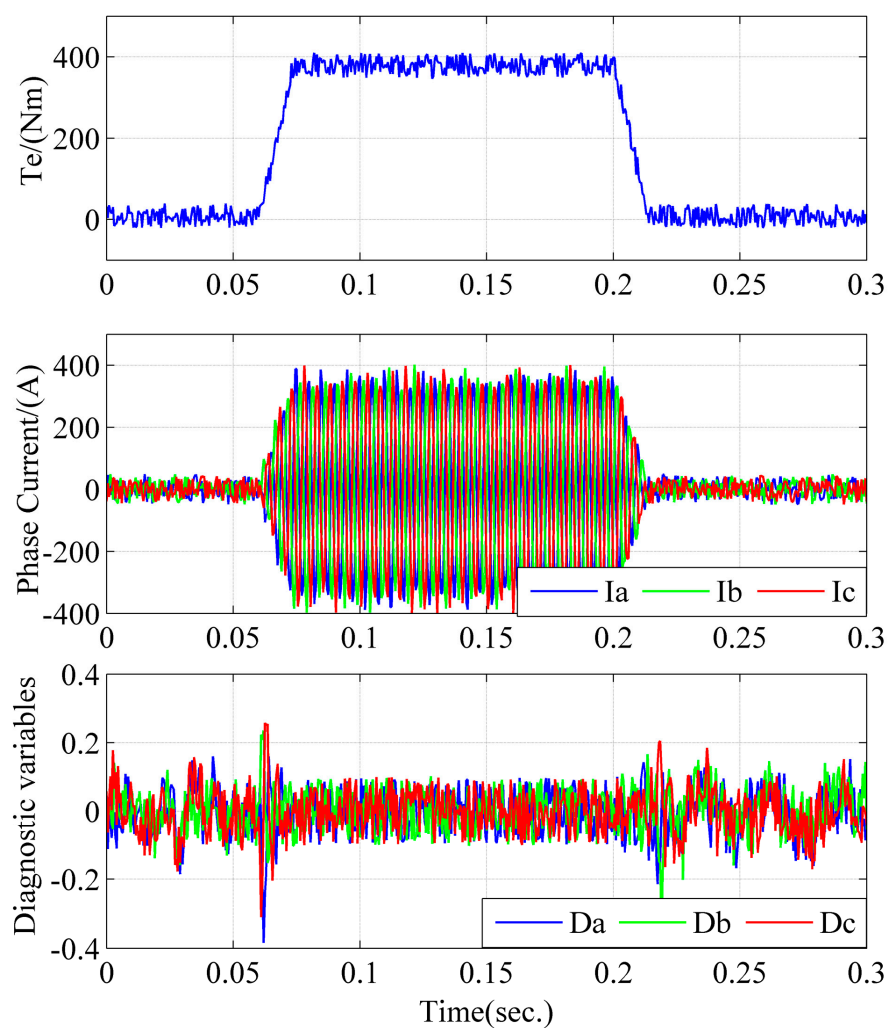

Figure 15. Experimental results under a sudden change of load torque conditions. 
Table 3 summarizes the detection speed results, showing the minimum, average, and maximum detection times achieved by the different algorithms. The modified normalized DC current algorithm is selected to compare with the proposed algorithm in this paper. Among the open-circuit fault algorithms, the modified normalized DC current method is found to be very effective in detecting faults with a high resistivity to false alarms. In addition, it is independent of the load variations [21]. Specifically, the minimum, average, and maximum detection times of the proposed algorithm are calculated according to the experimental results at $600 \mathrm{rpm} / 358 \mathrm{Nm}$.

As shown in Table 3, the proposed algorithm presents a much faster detection speed than the modified normalized DC current algorithm.

Table 3. Comparison of the detection speed.

\begin{tabular}{cccc}
\hline \multirow{2}{*}{ Diagnostic Algorithm } & \multicolumn{3}{c}{ Detection Speed (\% Current Period) } \\
\cline { 2 - 4 } & Min & Average & Max \\
\hline Modified Normalized & 33 & 79.5 & 125 \\
DC current [14] & 14.9 & 36.2 & 64.9 \\
Proposed Algorithm & 14.9 & \\
\hline
\end{tabular}

\section{Conclusions}

In this paper, a fast-acting diagnostic algorithm of IGBT open circuit faults for power inverters in electric vehicles is presented. The common open circuit faults of an inverter are analyzed. When an open circuit fault occurs, the half-bridge of the inverter can only output positive or negative current, resulting in the difference between the actual three-phase current and the referential three-phase current values. Based on this difference, the diagnostic algorithm for open circuit faults is proposed, combined with the normalization method and the VPMAM.

The effectiveness of the algorithm is verified by both simulations and experiments. This algorithm is robust, independent of load variations, and has a high resistivity against false alarms. Since only the three-phase current of the motor is utilized for calculations in the time domain, the algorithm needs less implementation effort.

Compared with other similar diagnostic algorithms, the proposed algorithm is much faster. The results show that the minimum detection speed of the proposed algorithm can be $14.9 \%$ of the motor phase current fundamental period, which is essential for real-time control in electric vehicles.

Author Contributions: This paper is the result of the hard work of all the authors. Lei Yu and Wenqing Huang conceived and designed the proposed method. Khaled Teffah, Lei Yu, and Youtong Zhang conceived and performed the experiments; Wenqing Huang and Lei Yu analyzed the data; Lei Yu wrote the paper. Khaled Teffah revised the English writing of the paper. All authors gave advice for the manuscript.

Conflicts of Interest: The authors declare no conflict of interest.

\section{References}

1. Chan, C.C.; Bouscayrol, A.; Chen, K. Electrical, hybrid, and fuel-cell vehicles: Architectures and modeling. IEEE Trans. Veh. Technol. 2010, 59, 589-598. [CrossRef]

2. Zhu, Z.Q.; Howe, D. Electricalal machines and drives for electrical, hybrid and fuel cell vehicles. Proc. IEEE 2007, 95, 746-765. [CrossRef]

3. Henao, H.; Capolino, G.A.; Fernandez-Cabanas, M.; Filippetti, F. Trends in fault diagnosis for electrical machines: A review of diagnostic techniques. IEEE Ind. Electron. Mag. 2014, 8, 31-42. [CrossRef]

4. Zhang, L.; Hu, Y.; Huang, W. Fault diagnosis and tolerant techniques of inverter in three-phase variable frequency drive system. Trans. Chin. Electrotech. Soc. 2004, 19, 1-5.

5. Jacobina, C.B.; de Araujo Ribeiro, R.L.; Lima, A.M.N.; da Silva, E.R.C. Fault-tolerant reversible AC motor drive system. IEEE Trans. Ind. Appl. 2003, 39, 1077-1084. [CrossRef] 
6. Karimi, S.; Gaillard, A.; Poure, P.; Saadate, S. FPGA-based real-time power converter failure diagnosis for wind energy conversion systems. IEEE Trans. Ind. Electron. 2009, 55, 4299-4308. [CrossRef]

7. An, Q.T.; Sun, L.; Zhao, K.; Liu, C.; Yu, L.N. Diagnosis method for inverter open circuit fault based on switching function model. Proc. CSEE 2010, 30, 1-6.

8. Shahbazi, M.; Zolghadri, M.R.; Poure, P.; Saadate, S. Fast detection of open-switch faults with reduced sensor count for a fault-tolerant three-phase converter. In Proceedings of the Power Electronics, Drive Systems and Technologies Conference, Tehran, Iran, 16-17 February 2011; pp. 546-550.

9. Estima, J.O.; Cardoso, A.J.M. A new algorithm for real-time multiple open circuit fault diagnosis in voltage-fed PWM motor drives by the reference current errors. IEEE Trans. Ind. Electron. 2012, 60, 3496-3505. [CrossRef]

10. An, Q.; Sun, L.; Zhao, K. Recent developments of fault diagnosis methods for switches in three-phase inverters. Trans. Chin. Electrotech. Soc. 2011, 26, 135-144.

11. Mendes, A.M.S.; Cardoso, A.J.M.; Saraiva, E.S. Voltage source inverter fault diagnosis in variable speed AC drives, by Park's vector approach. In Proceedings of the International Conference on Electrical Machines and Drives, Canterbury, UK, 1-3 September 1999; pp. 704-706.

12. Peuget, R.; Courtine, S.; Rognon, J.P. Fault detection and isolation on a PWM inverter by knowledge-based model. In Proceedings of the 1997 IEEE International Conference on Control Applications, Hartford, CT, USA, 5-7 October 1997; pp. 565-570.

13. Abramik, S.; Sleszynski, W.; Nieznanski, J.; Piquet, H. A diagnostic method for on-line fault detection and localization in VSI-fed AC drives. In Proceedings of the 2003 European Conference on Power Electronics and Applications, Toulouse, France, 2-4 September 2003; pp. 2-4.

14. Rothenhagen, K.; Fuchs, F.W. Performance of Diagnosis Methods for IGBT Open Circuit Faults in Three Phase Voltage Source Inverters for AC Variable Speed Drives. In Proceedings of the 2005 European Conference on Power Electronics and Applications, Dresden, Germany, 11-14 September 2005; pp. 10-17.

15. Baek, S.K.; Shin, H.U.; Kang, S.Y.; Park, C.S.; Lee, K.B. Open fault detection and tolerant control for a five phase inverter driving system. Energies 2016, 9, 355. [CrossRef]

16. Qiu, Y.; Jiang, H.; Feng, Y.; Cao, M.; Zhao, Y.; Li, D. A new fault diagnosis algorithm for PMSG wind turbine power converters under variable wind speed conditions. Energies 2016, 9, 548. [CrossRef]

17. Jamshidpour, E.; Shahbazi, M.; Saadate, S.; Poure, P.; Gholipour, E. FPGA based fault detection and fault tolerance operation in DC-DC converters. In Proceedings of the 2014 International Symposium on Power Electronics, Electrical Drives, Automation and Motion (SPEEDAM), Ischia, Italy, 18-20 June 2014; pp. 37-42.

18. Park, J.H.; Kim, D.H.; Kim, S.S.; Lee, D.J. C-ANFIS based fault diagnosis for voltage-fed PWM motor drive systems. In Proceedings of the IEEE Meeting of the Fuzzy Information Processing NAFIPS ‘04, Banff, AB, Canada, 27-30 June 2004; Volume 371, pp. 379-383.

19. Zidani, F.; Diallo, D.; Benbouzid, M.E.H.; Nait-Said, R. A fuzzy-based approach for the diagnosis of fault modes in a voltage-fed PWM inverter induction motor drive. IEEE Trans. Ind. Electron. 2008, 55, 586-593. [CrossRef]

20. Charfi, F.; Sellami, F.; Al-Haddad, K. Fault Diagnostic in Power System Using Wavelet Transforms and Neural Networks. In Proceedings of the 2006 IEEE International Symposium on Industrial Electronics, Montreal, QC, Canada, 9-13 July 2006; pp. 1143-1148.

21. Lu, B.; Sharma, S.K. A literature review of IGBT fault diagnostic and protection methods for power inverters. IEEE Trans. Ind. Appl. 2008, 45, 1770-1777.

22. Zhao, J.; Gao, X.; Li, B.; Liu, X.; Guan, X. Open-phase fault tolerance techniques of five-phase dual-rotor permanent magnet synchronous motor. Energies 2015, 8, 12810-12838. [CrossRef]

23. Huang, W.; Zhang, Y.; Zhang, X.; Sun, G. Accurate torque control of interior permanent magnet synchronous machine. IEEE Trans. Energy Convers. 2014, 29, 29-37. [CrossRef]

24. Estima, J.O.; Freire, N.M.A.; Cardoso, A.J.M. Recent Advances in Fault Diagnosis by Park's Vector Approach. In Proceedings of the 2013 IEEE Workshop on Electrical Machines Design Control and Diagnosis, Paris, France, 11-12 March 2013; pp. 279-288.

(C) 2017 by the authors. Licensee MDPI, Basel, Switzerland. This article is an open access article distributed under the terms and conditions of the Creative Commons Attribution (CC BY) license (http:/ / creativecommons.org/licenses/by/4.0/). 